\title{
Article \\ A Comparative Analysis of Fractional-Order Gas Dynamics Equations via Analytical Techniques
}

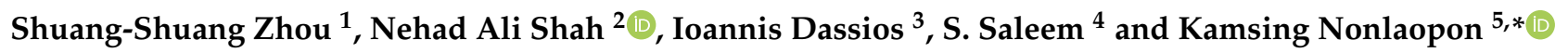 \\ 1 College of Science, Hunan City University, Yiyang 413000, China; zhoushuangshuang21@126.com \\ 2 Department of Mechanical Engineering, Sejong University, Seoul 05006, Korea; nehadali199@yahoo.com \\ 3 AMPSAS, University College Dublin, D04 V1W8 Dublin, Ireland; ioannis.dassios@ucd.ie \\ 4 Department of Mathematics, College of Science, King Khalid University, Abha 61413, Saudi Arabia; \\ saakhtar@kku.edu.sa \\ 5 Department of Mathematics, Faculty of Science, Khon Kaen University, Khon Kaen 40002, Thailand \\ * Correspondence: nkamsi@kku.ac.th
}

Citation: Zhou, S.-S.; Shah, N.A.;

Dassios, I.; Saleem, S.; Nonlaopon, K. A Comparative Analysis of Fractional-Order Gas Dynamics Equations via Analytical Techniques. Mathematics 2021, 9, 1735. https:// doi.org/10.3390/math9151735

Academic Editor: Mariano Torrisi

Received: 17 May 2021

Accepted: 17 July 2021

Published: 22 July 2021

Publisher's Note: MDPI stays neutral with regard to jurisdictional claims in published maps and institutional affiliations.

Copyright: (c) 2021 by the authors. Licensee MDPI, Basel, Switzerland. This article is an open access article distributed under the terms and conditions of the Creative Commons Attribution (CC BY) license (https:// creativecommons.org/licenses/by/ $4.0 /)$.

\begin{abstract}
This article introduces two well-known computational techniques for solving the timefractional system of nonlinear equations of unsteady flow of a polytropic gas. The methods suggested are the modified forms of the variational iteration method and the homotopy perturbation method by the Elzaki transformation. Furthermore, an illustrative scheme is introduced to verify the accuracy of the available techniques. A graphical representation of the exact and derived results is presented to show the reliability of the suggested approaches. It is also shown that the findings of the current methodology are in close harmony with the exact solutions. The comparative solution analysis via graphs also represents the higher reliability and accuracy of the current techniques.
\end{abstract}

Keywords: Elzaki transform; homotopy perturbation method; variational iteration method; gas dynamic equations; Mittag-Leffler function

\section{Introduction}

Gas dynamic equations are mathematical representations based on physical conservation laws such as the conservation of energy, the conservation of mass laws, the conservation of momentum, etc. The nonlinear equations of ideal gas dynamics are implemented regarding three types of nonlinear waves, such as contact discontinuities shock fronts, and refractions-see [1]. In 1981, Steger and Warming [2] proposed that the conservation-law model of the inviscid gas dynamic equation possess interests by which nonlinear flux vectors are homogeneous degree one functions allowing the division of flux vectors into sub-vectors through similarity transforms; as a result, new explicit and implicit finite-difference dissipative systems for analyzing first-order hyperbolic equation schemes were created. In terms of developing an appropriate flow method for solving a slowly changing gas dynamic system under the gravity field, the numerical scheme must be well-balanced [3-6]. For instance, most astrophysical models are related to hydrodynamic evolution in the gravitational area; the helpful application of the gravitational force in the astronomical hydrodynamic code is essential to capture the long-term development of star simulation and solar system formation. Although several hydrodynamic modules were effectively implemented to solve astrophysical issues, such as the piecewise Parabolic technique and total variation diminishing codes $[7,8]$, most considered only short-term changes with robust expansion or shock waves. With the slowness of the evolution of the galaxy, the different codes have significant drawbacks to the improper usage of the gravitational potential effect, the so-called source term in the Euler or Navier-Stokes equations [9]. 
In the present study, we consider the gas dynamic equations fractional-order scheme describing the production of the unsteady two-dimensional streaming of the ideal fluid. In astrophysics, polytropic gas is defined by [10]

$$
\psi=k \omega^{1+\frac{1}{m}}
$$

where $\psi=\frac{\theta}{\phi}$ is the energy density, $\phi$ is the container volume, $\theta$ is the total the energy of the gas, $m$ is the polytropic index and $k$ is a constant. Degenerate adiabatic gas and electron gas are two instances of such gases. In astrophysics and cosmology, the analysis of polytropic gases plays a critical role and these gases can behave like dark energy [11]. Now consider the gas dynamic equations scheme, which describes the evolution of unstable flow of a perfect gas with fractional derivatives [10,12]:

$$
\begin{array}{cc}
D_{\Im}^{\sigma} \mu+\mu \frac{\partial \mu}{\partial \varphi}+v \frac{\partial \mu}{\partial \vartheta}+\frac{1}{\omega} \frac{\partial \psi}{\partial \varphi}=0, & D_{\Im}^{\sigma} v+\mu \frac{\partial v}{\partial \varphi}+v \frac{\partial v}{\partial \vartheta}+\frac{1}{\omega} \frac{\partial \psi}{\partial \vartheta}=0, \\
D_{\Im}^{\sigma} \omega+\mu \frac{\partial \omega}{\partial \varphi}+v \frac{\partial \omega}{\partial \vartheta}+\omega\left(\frac{\partial \mu}{\partial \varphi}+\frac{\partial v}{\partial \vartheta}\right)=0, & D_{\Im}^{\sigma} \psi+\mu \frac{\partial \psi}{\partial \varphi}+v \frac{\partial \psi}{\partial \vartheta}+\eta \psi\left(\frac{\partial \mu}{\partial \varphi}+\frac{\partial v}{\partial \vartheta}\right)=0,
\end{array}
$$

with initial conditions

$$
\begin{aligned}
& \mu(\varphi, \vartheta, 0)=\alpha(\varphi+\vartheta), \quad v(\varphi, \vartheta, 0)=\beta(\varphi+\vartheta), \\
& \omega(\varphi, \vartheta, 0)=\gamma(\varphi+\vartheta), \quad \psi(\varphi, \vartheta, 0)=\Phi(\varphi+\vartheta) .
\end{aligned}
$$

where $\mu(\varphi, \vartheta, \Im)$ and $v(\varphi, \vartheta, \Im)$ are the velocity components, $\omega(\varphi, \vartheta, \Im)$ is the density, $\psi(\varphi, \vartheta, \Im)$ is the pressure and $\Im$ is the ratio of the specific heat and it represents the adiabatic index. In the past decade, the approximate analytical solutions of different types of gas have been achieved by many numerical and analytical techniques. Various techniques has been solved by gas dynamics problems, such as the fractional reduced differential transform technique [13], homotopy perturbation technique [14], q-homotopy analysis technique [10], Adomian decomposition technique [15], fractional homotopy analysis transform technique [16] and natural decomposition technique [17].

The variational iteration method (VIM) was first introduced by J.He [13] and effectively implemented in the treatment of heat conduction models [14-17]. In 2010, a fractional variational iteration method (FVIM) was developed using a modified Riemann-Liouville derivative [17]. The classification of the Lagrange multiplier plays an essential role for the variational iteration method, and the variational concept is commonly used for this aim. A more straightforward approach to the Elzaki of transformation to determine the multiplier is proposed, making the technique available to researchers with different nonlinear problems. The variational iteration method combines with other transformations and has been solved in many papers, such as the work of Abassy et al., who implemented the VIM with the Laplace transformation in 2007 [12], as did Hesameddini and Latifizadeh 2009 [14] and Mokhtari and Mohammadi 2009 [13]. Abassy, El-Tawil and El-Zoheiry [12] used the Laplace transformation in the solution process; the variational iteration method led to a series of linear equations, which can be easily solved by the Laplace transformation.

The homotopy perturbation method (HPM) was first introduced by the Chinese mathematician J.H. He played a key role in this research in 1998 [18]. This method is efficient, accurate, and effective and eliminates infinite series, an unconditioned matrix, and difficult integrals. This methodology does not require a specific parameter of the equation. In this method, according to the homotopy technique, a homotopy with an embedding parameter $p \in[0,11]$ is developed and the encoding parameter is called a "small parameter", which can ensure the full benefits of the conventional perturbation techniques and homotopy techniques [19-22]. The Elzaki transformation (E.T.) is a new integral transform introduced by Tarig Elzaki in 2010. The E.T. is a modified transform of Sumudu and Laplace transforms. It is important to remember that there are certain mathematical models with variable coefficients that Sumudu and Laplace transforms 
cannot be used for but can be conveniently carried out by using the E.T. [23-25]. The homotopy perturbation transform method is a combination of the Elzaki transformation and homotopy perturbation technique. Many researchers have solved different equations with the help of HPETM, such as Navier-Stokes equations [26], heat-like equations [27], Fisher's equation and hyperbolic equations [28].

In this paper, the HPTM and VITM are implemented to analyze the fractional-order gas dynamic equation. The HPTM and VITM solutions are determined for the particular model of the fractional-order two-dimensional gas dynamic equation. The higher accuracy and efficiency of HPTM and VITM are investigated, and the analysis of graphs is compared to actual solutions. The series form results of HPTM and VITM for fractional-order gas dynamic equations has shown the desire rate of convergence. Thus, the current techniques are suggested to solve other fractional-order linear and nonlinear partial differential equations.

\section{Basic Preliminaries}

Definition 1. The operator $D^{\sigma}$ of order $\sigma$ in Abel-Riemann sense is given as [23-25]

$$
D^{\sigma} v(\varphi)=\left\{\begin{array}{l}
\frac{d^{j}}{d \varphi^{j}} v(\varphi), \sigma=j \\
\frac{1}{\Gamma(j-\sigma)} \frac{d}{d \varphi^{j}} \int_{0}^{\varphi} \frac{v(\varphi)}{(\varphi-\psi)^{\sigma-j+1}} d \psi, j-1<\sigma<j
\end{array}\right.
$$

where $j \in Z^{+}, \sigma \in R^{+}$and

$$
D^{-\sigma} v(\varphi)=\frac{1}{\Gamma(\sigma)} \int_{0}^{\varphi}(\varphi-\psi)^{\sigma-1} v(\psi) d \psi, 0<\sigma \leq 1 .
$$

Definition 2. The Abel-Riemann fractional-order integration operator $J^{\sigma}$ is defined as [23-25]

$$
J^{\sigma} v(\varphi)=\frac{1}{\Gamma(\sigma)} \int_{0}^{\varphi}(\varphi-\psi)^{\sigma-1} v(\varphi) d \varphi, \varphi>0, \sigma>0 .
$$

The operator of basic properties:

$$
\begin{aligned}
J^{\sigma} \varphi^{j} & =\frac{\Gamma(j+1)}{\Gamma(j+\sigma+1)} \varphi^{j+\sigma} \\
D^{\sigma} \varphi^{j} & =\frac{\Gamma(j+1)}{\Gamma(j-\sigma+1)} \varphi^{j-\sigma}
\end{aligned}
$$

Definition 3. The Caputo fractional operator $D^{\sigma}$ of $\rho$ is defined as [23-25]

$$
{ }^{C} D^{\sigma} v(\varphi)=\left\{\begin{array}{l}
\frac{1}{\Gamma(j-\sigma)} \int_{0}^{\varphi} \frac{v^{j}(\psi)}{(\varphi-\psi)^{\sigma-j+1}} d \psi, j-1<\sigma<j, \\
\frac{d^{j}}{d \varphi^{j}} v(\varphi), \quad j=\sigma .
\end{array}\right.
$$

Definition 4.

$$
\begin{aligned}
& J_{\varphi}^{\sigma} D_{\varphi}^{\sigma} g(\varphi)=g(\varphi)-\sum_{k=0}^{m} g^{k}\left(0^{+}\right) \frac{\varphi^{k}}{k !}, \text { for } \varphi>0, \text { and } j-1<\sigma \leq j, j \in N . \\
& D_{\varphi}^{\sigma} J_{\varphi}^{\sigma} g(\varphi)=g(\varphi) .
\end{aligned}
$$

Definition 5. The fractional-order Caputo operator of Elzaki transform is given as:

$$
E\left[D_{\varphi}^{\sigma} g(\varphi)\right]=s^{-\sigma} E[g(\varphi)]-\sum_{k=0}^{j-1} s^{2-\sigma+k} g^{(k)}(0), \text { where } j-1<\sigma<j .
$$




\section{The Technique of VITM}

In this section, discuss the VITM solution system for fractional partial differential equations.

$$
\begin{aligned}
& D_{\Im}^{\sigma} \mu(\varphi, \vartheta, \Im)+\mathcal{M}_{1}(\mu, v)+\mathcal{N}_{1}(\mu, v)-\mathcal{K}_{1}(\varphi, \vartheta, \Im)=0, \\
& D_{\Im}^{\sigma} v(\varphi, \vartheta, \Im)+\mathcal{M}_{2}(\mu, v)+\mathcal{N}_{2}(\mu, v)-\mathcal{K}_{2}(\varphi, \vartheta, \Im)=0, m-1<\sigma \leq m,
\end{aligned}
$$

with initial conditions

$$
\mu(\varphi, \vartheta, 0)=g_{1}(\varphi, \vartheta), \quad v(\varphi, \vartheta, 0)=g_{2}(\varphi, \vartheta) .
$$

where $D_{\Im}^{\sigma}=\frac{\partial^{\sigma}}{\partial \Im^{\sigma}}$ is the fractional-order Caputo operator of $\sigma, \mathcal{M}_{1}, \mathcal{M}_{2}$ and $\mathcal{N}_{1}, \mathcal{N}_{2}$ are linear and nonlinear functions, respectively, and $\mathcal{K}_{1}, \mathcal{K}_{2}$ are source terms.

The Elzaki transform is implemented to Equation (1), we get

$$
\begin{aligned}
& E\left[D_{\Im}^{\sigma} \mu(\varphi, \vartheta, \Im)\right]+E\left[\mathcal{M}_{1}(\mu, v)+\mathcal{N}_{1}(\mu, v)-\mathcal{K}_{1}(\varphi, \vartheta, \Im)\right]=0, \\
& E\left[D_{\Im}^{\sigma} v(\varphi, \vartheta, \Im)\right]+E\left[\mathcal{M}_{2}(\mu, v)+\mathcal{N}_{2}(\mu, v)-\mathcal{K}_{2}(\varphi, \vartheta, \Im)\right]=0,
\end{aligned}
$$

Implementing the differentiation property of the Elzaki Transform, we obtain

$$
\begin{aligned}
& E[\mu(\varphi, \vartheta, \Im)]-\left.\sum_{k=0}^{n-1}(s)^{2-\sigma+k} \frac{\partial^{k} \mu(\varphi, \vartheta, \Im)}{\partial^{k} \Im}\right|_{\Im=0}=-E\left[\mathcal{M}_{1}(\mu, v)+\mathcal{N}_{1}(\mu, v)-\mathcal{K}_{1}(\varphi, \vartheta, \Im)\right], \\
& E[v(\varphi, \vartheta, \Im)]-\left.\sum_{k=0}^{n-1}(s)^{2-\sigma+k} \frac{\partial^{k} v(\varphi, \vartheta, \Im)}{\partial^{k} \Im}\right|_{\Im=0}=-E\left[\mathcal{M}_{2}(\mu, v)+\mathcal{N}_{2}(\mu, v)-\mathcal{K}_{2}(\varphi, \vartheta, \Im)\right], \\
& E\left[\mu_{m+1}(\varphi, \vartheta, \Im)\right]=E\left[\mu_{m}(\varphi, \vartheta, \Im)\right]+\lambda(s)\left[\frac{1}{s^{\sigma}} \mu_{m}(\varphi, \vartheta, \Im)-\left.\sum_{k=0}^{n-1}(s)^{2-\sigma+k} \frac{\partial^{k} \mu(\varphi, \vartheta, \Im)}{\partial^{k} \Im}\right|_{\Im=0}\right. \\
& \left.-E\left[\mathcal{K}_{1}(\varphi, \vartheta, \Im)\right]-E\left\{\mathcal{M}_{1}(\mu, v)+\mathcal{N}_{1}(\mu, v)\right\}\right], \\
& E\left[v_{m+1}(\varphi, \vartheta, \Im)\right]=E\left[v_{m}(\varphi, \vartheta, \Im)\right]+\lambda(s)\left[\frac{1}{s^{\sigma}} v_{m}(\varphi, \vartheta, \Im)-\left.\sum_{k=0}^{n-1}(s)^{2-\sigma+k} \frac{\partial^{k} v(\varphi, \vartheta, \Im)}{\partial^{k} \Im}\right|_{\Im=0}\right. \\
& \left.-E\left[\mathcal{K}_{2}(\varphi, \vartheta, \Im)\right]-E\left\{\mathcal{M}_{2}(\mu, v)+\mathcal{N}_{2}(\mu, v)\right\}\right],
\end{aligned}
$$

A Lagrange multiplier as

$$
\lambda(s)=-s^{\sigma},
$$

Applying inverse Elzaki transform $E^{-1}$ to Equation (5)

$$
\begin{aligned}
\mu_{m+1}(\varphi, \vartheta, \Im)= & \mu_{m}(\varphi, \vartheta, \Im)-E^{-1}\left[s ^ { \sigma } \left[\left.\sum_{k=0}^{n-1}(s)^{2-\sigma+k} \frac{\partial^{k} \mu(\varphi, \vartheta, \Im)}{\partial^{k} \Im}\right|_{\Im=0}\right.\right. \\
& \left.\left.-E\left[\mathcal{K}_{1}(\varphi, \vartheta, \Im)\right]-E\left\{\mathcal{M}_{1}(\mu, v)+\mathcal{N}_{1}(\mu, v)\right\}\right]\right], \\
v_{m+1}(\varphi, \vartheta, \Im)= & v_{m}(\varphi, \vartheta, \Im)-E^{-1}\left[s ^ { \sigma } \left[\left.\sum_{k=0}^{n-1}(s)^{2-\sigma+k} \frac{\partial^{k} v(\varphi, \vartheta, \Im)}{\partial^{k} \Im}\right|_{\Im=0}\right.\right. \\
& \left.\left.-E\left[\mathcal{K}_{2}(\varphi, \vartheta, \Im)\right]-E\left\{\mathcal{M}_{2}(\mu, v)+\mathcal{N}_{2}(\mu, v)\right\}\right]\right],
\end{aligned}
$$

The convergence of this technique is shown in $[29,30]$.

\section{The General Methodology of HPTM}

The general methodology of the given method:

$$
\begin{aligned}
& D_{\Im}^{\sigma} \mu(\varphi, \vartheta)+M \mu(\varphi, \vartheta, \Im)+N \mu(\varphi, \vartheta, \Im)=h(\varphi, \vartheta, \Im), \quad \vartheta>0, \quad 0<\sigma \leq 1, \\
& \mu(\varphi, 0)=g(\chi), \quad v \in \Re .
\end{aligned}
$$


Using Elzaki transform of Equation (8), we obtain

$$
\begin{aligned}
& E\left[D_{\Im}^{\sigma} \mu(\varphi, \vartheta, \Im)+M \mu(\varphi, \vartheta, \Im)+N \mu(\varphi, \vartheta, \Im)\right]=E[h(\varphi, \vartheta, \Im)], \quad \vartheta>0, \quad 0<\sigma \leq 1, \\
& \mu(\varphi, \vartheta, \Im)=s^{2} g(\varphi)+s^{\sigma} E[h(\varphi, \vartheta, \Im)]-s^{\sigma} E[M \mu(\varphi, \vartheta, \Im)+N \mu(\varphi, \vartheta, \Im)] .
\end{aligned}
$$

Now, by using an inverse Elzaki transform

$$
\mu(\varphi, \vartheta, \Im)=F(\varphi, \vartheta)-E^{-1}\left[s^{\sigma} E\{M \mu(\varphi, \vartheta, \Im)+N \mu(\varphi, \vartheta, \Im)\}\right],
$$

where

$$
F(\varphi, \vartheta, \Im)=E^{-1}\left[s^{2} g(\varphi)+s^{\sigma} E[h(\varphi, \vartheta, \Im)]\right]=g(v)+E^{-1}\left[s^{\sigma} E[h(\varphi, \vartheta, \Im)]\right] .
$$

Now, perturbation method is presented as

$$
\mu(\varphi, \vartheta, \Im)=\sum_{k=0}^{\infty} p^{k} \mu_{k}(\varphi, \vartheta, \Im),
$$

where the perturbation parameter is $p$ and $p \in[0,1]$.

The nonlinear terms can be explain as

$$
N \mu(\varphi, \vartheta, \Im)=\sum_{k=0}^{\infty} p^{k} H_{k}\left(\mu_{k}\right),
$$

where $H_{n}$ are He's polynomials in terms of $\mu_{0}, \mu_{1}, \mu_{2}, \ldots, \mu_{n}$, and can be determined as

$$
H_{n}\left(\psi_{0}, \psi_{1}, \cdots, \psi_{n}\right)=\frac{1}{n !} \frac{\partial^{n}}{\partial p^{n}}\left[N\left(\sum_{k=0}^{\infty} p^{k} \psi_{k}\right)\right]_{p=0}, m=0,1,2 \cdots
$$

Putting Equations (13) and (14) in Equation (10), we obtain

$$
\sum_{k=0}^{\infty} p^{k} \mu_{k}(\varphi, \vartheta, \Im)=F(\varphi, \vartheta, \Im)-p \times\left[E^{-1}\left\{s^{\sigma} E\left\{M \sum_{k=0}^{\infty} p^{k} \mu_{k}(\varphi, \vartheta, \Im)+\sum_{k=0}^{\infty} p^{k} H_{k}\left(\mu_{k}\right)\right\}\right\}\right] \text {. }
$$

Comparing the coefficient of equal powers of $p$ from both sides of above equation, the following equations are achieved

$$
\begin{aligned}
& p^{0}: \mu_{0}(\varphi, \vartheta, \Im)=F(\varphi, \vartheta, \Im), \\
& p^{1}: \mu_{1}(\varphi, \vartheta, \Im)=E^{-1}\left[s^{\sigma} E\left(M \mu_{0}(\varphi, \vartheta, \Im)+H_{0}(\mu)\right)\right], \\
& p^{2}: \mu_{2}(\varphi, \vartheta, \Im)=E^{-1}\left[s^{\sigma} E\left(M \mu_{1}(\varphi, \vartheta, \Im)+H_{1}(\mu)\right)\right], \\
& \vdots \\
& p^{k}: \mu_{k}(\varphi, \vartheta, \Im)=E^{-1}\left[s^{\sigma} E\left(M \mu_{k-1}(\varphi, \vartheta, \Im)+H_{k-1}(\mu)\right)\right], \quad k>0, k \in N .
\end{aligned}
$$

The solution is written as

$$
\mu(\varphi, \vartheta, \Im)=\mu_{1}(\varphi, \vartheta, \Im)+\mu_{2}(\varphi, \vartheta, \Im)+\mu_{3}(\varphi, \vartheta, \Im)+\cdots .
$$




\section{Applications}

Example 1. Consider the fractional-order nonlinear system gas equations

$$
\begin{aligned}
& D_{\Im}^{\sigma} \mu+\mu \frac{\partial \mu}{\partial \varphi}+v \frac{\partial \mu}{\partial \vartheta}+\frac{1}{\omega} \frac{\partial \psi}{\partial \varphi}=0, \\
& D_{\Im}^{\sigma} \nu+\mu \frac{\partial v}{\partial \varphi}+v \frac{\partial v}{\partial \vartheta}+\frac{1}{\omega} \frac{\partial \psi}{\partial \vartheta}=0, \\
& D_{\Im}^{\sigma} \omega+\mu \frac{\partial \omega}{\partial \varphi}+v \frac{\partial \omega}{\partial \vartheta}+\omega\left(\frac{\partial \mu}{\partial \varphi}+\frac{\partial v}{\partial \vartheta}\right)=0, \\
& D_{\Im}^{\sigma} \psi+\mu \frac{\partial \psi}{\partial \varphi}+v \frac{\partial \psi}{\partial \vartheta}+\Im \psi\left(\frac{\partial \mu}{\partial \varphi}+\frac{\partial v}{\partial \vartheta}\right)=0,
\end{aligned}
$$

with the initial conditions

$$
\begin{aligned}
& \mu(\varphi, \vartheta, 0)=e^{\varphi+\vartheta}, \quad \nu(\varphi, \vartheta, 0)=-1-e^{\varphi+\vartheta}, \\
& \omega(\varphi, \vartheta, 0)=e^{\varphi+\vartheta}, \quad \psi(\varphi, \vartheta, 0)=c .
\end{aligned}
$$

where $c$ is the real constant. First, we solve this system with the help of HPTM. Now, using the Elzaki transform of Equation (18), we obtain

$$
\begin{gathered}
\frac{1}{s^{\sigma}} E[\mu(\varphi, \vartheta, \Im)]=\mu(\varphi, \vartheta, 0) s^{2-\sigma}-E\left\{\mu \frac{\partial \mu}{\partial \varphi}+v \frac{\partial \mu}{\partial \vartheta}+\frac{1}{\omega} \frac{\partial \psi}{\partial \varphi}\right\}, \\
\frac{1}{s^{\sigma}} E[\nu(\varphi, \vartheta, \Im)]=\nu(\varphi, \vartheta, 0) s^{\sigma+2}-E\left\{\mu \frac{\partial v}{\partial \varphi}+v \frac{\partial v}{\partial \vartheta}+\frac{1}{\omega} \frac{\partial \psi}{\partial \vartheta}\right\}, \\
\frac{1}{s^{\sigma}} E[\omega(\varphi, \vartheta, \Im)]=\omega(\varphi, \vartheta, 0) s^{2-\sigma}-E\left\{\mu \frac{\partial \omega}{\partial \varphi}+v \frac{\partial \omega}{\partial \vartheta}+\omega\left(\frac{\partial \mu}{\partial \varphi}+\frac{\partial v}{\partial \vartheta}\right)\right\}, \\
\frac{1}{s^{\sigma}} E[\psi(\varphi, \vartheta, \Im)]=\psi(\varphi, \vartheta, 0) s^{\sigma+2}-E\left\{\mu \frac{\partial \psi}{\partial \varphi}+v \frac{\partial \psi}{\partial \vartheta}+\Im \psi\left(\frac{\partial \mu}{\partial \varphi}+\frac{\partial v}{\partial \vartheta}\right)\right\}, \\
E[\mu(\varphi, \vartheta, \Im)]=s^{2} \mu(\varphi, \vartheta, 0)-s^{\sigma} E\left\{\mu \frac{\partial \mu}{\partial \varphi}+v \frac{\partial \mu}{\partial \vartheta}+\frac{1}{\omega} \frac{\partial \psi}{\partial \varphi}\right\}, \\
E[v(\varphi, \vartheta, \Im)]=s^{2} \mu(\varphi, \vartheta, 0)-s^{\sigma} E\left\{\mu \frac{\partial v}{\partial \varphi}+v \frac{\partial v}{\partial \vartheta}+\frac{1}{\omega} \frac{\partial \psi}{\partial \vartheta}\right\}, \\
E[\omega(\varphi, \vartheta, \Im)]=s^{2} \mu(\varphi, \vartheta, 0)-s^{\sigma} E\left\{\mu \frac{\partial \omega}{\partial \varphi}+v \frac{\partial \omega}{\partial \vartheta}+\omega\left(\frac{\partial \mu}{\partial \varphi}+\frac{\partial v}{\partial \vartheta}\right)\right\}, \\
E[\psi(\varphi, \vartheta, \Im)]=s^{2} \mu(\varphi, \vartheta, 0)-s^{\sigma} E\left\{\mu \frac{\partial \psi}{\partial \varphi}+v \frac{\partial \psi}{\partial \vartheta}+\Im \psi\left(\frac{\partial \mu}{\partial \varphi}+\frac{\partial v}{\partial \vartheta}\right)\right\} .
\end{gathered}
$$

Applying the inverse Elzaki transform, we obtain

$$
\begin{aligned}
& E[\mu(\varphi, \vartheta, \Im)]=e^{\varphi+\vartheta}-E^{-1}\left[s^{\sigma} E\left\{\mu \frac{\partial \mu}{\partial \varphi}+v \frac{\partial \mu}{\partial \vartheta}+\frac{1}{\omega} \frac{\partial \psi}{\partial \varphi}\right\}\right], \\
& E[\nu(\varphi, \vartheta, \Im)]=-1-e^{\varphi+\vartheta}-E^{-1}\left[s^{\sigma} E\left\{\mu \frac{\partial v}{\partial \varphi}+v \frac{\partial v}{\partial \vartheta}+\frac{1}{\omega} \frac{\partial \psi}{\partial \vartheta}\right\}\right], \\
& E[\omega(\varphi, \vartheta, \Im)]=e^{\varphi+\vartheta}-E^{-1}\left[s^{\sigma} E\left\{\mu \frac{\partial \omega}{\partial \varphi}+v \frac{\partial \omega}{\partial \vartheta}+\omega\left(\frac{\partial \mu}{\partial \varphi}+\frac{\partial v}{\partial \vartheta}\right)\right\}\right], \\
& E[\psi(\varphi, \vartheta, \Im)]=c-E^{-1}\left[s^{\sigma} E\left\{\mu \frac{\partial \psi}{\partial \varphi}+v \frac{\partial \psi}{\partial \vartheta}+\Im \psi\left(\frac{\partial \mu}{\partial \varphi}+\frac{\partial v}{\partial \vartheta}\right)\right\}\right] .
\end{aligned}
$$


The HPM in Equation (22), we can archive this as

$$
\begin{aligned}
& \sum_{\kappa=0}^{\infty} p^{\kappa} \mu_{\kappa}(\varphi, \vartheta)=e^{\varphi+\vartheta}-p\left[E^{-1}\left\{s^{\sigma} E\left\{\sum_{\kappa=0}^{\infty} p^{\kappa} H_{\kappa}(\mu)\right\}\right\},\right. \\
& \sum_{\kappa=0}^{\infty} p^{\kappa} v_{\mathcal{K}}(\varphi, \vartheta)=-1-e^{\varphi+\vartheta}-p\left[E^{-1}\left\{s^{\sigma} E\left\{\sum_{\kappa=0}^{\infty} p^{\kappa} H_{\mathcal{K}}(v)\right\}\right\},\right. \\
& \sum_{\kappa=0}^{\infty} p^{\kappa} \omega_{\mathcal{K}}(\varphi, \vartheta)=e^{\varphi+\vartheta}-p\left[E^{-1}\left\{s^{\sigma} E\left\{\sum_{\kappa=0}^{\infty} p^{\kappa} H_{\kappa}(\omega)\right\}\right\}\right] \\
& \sum_{\kappa=0}^{\infty} p^{\kappa} \psi_{\kappa}(\varphi, \vartheta)=c-p\left[E^{-1}\left\{s^{\sigma} E\left\{\sum_{\kappa=0}^{\infty} p^{\kappa} H_{\mathcal{K}}(\psi)\right\}\right\}\right]
\end{aligned}
$$

where $H_{\mathcal{K}}(\mu), H_{\mathcal{K}}(v), H_{\mathcal{K}}(\omega)$ and $H_{\mathcal{K}}(\psi)$ are He's polynomials, which signify the nonlinear terms. The first few terms of He's polynomials are suggested as

$$
\begin{gathered}
H_{0}(\mu)=\mu_{0} \frac{\partial \mu_{0}}{\partial \varphi}+v_{0} \frac{\partial \mu_{0}}{\partial \vartheta}+\frac{1}{\omega_{0}} \frac{\partial \psi_{0}}{\partial \varphi} \\
H_{0}(v)=\mu_{0} \frac{\partial v_{0}}{\partial \varphi}+v_{0} \frac{\partial v_{0}}{\partial \vartheta}+\frac{1}{\omega_{0}} \frac{\partial \psi_{0}}{\partial \vartheta} \\
H_{0}(\omega)=\mu_{0} \frac{\partial \omega_{0}}{\partial \varphi}+v_{0} \frac{\partial \omega_{0}}{\partial \vartheta}+\omega_{0}\left(\frac{\partial \mu_{0}}{\partial \varphi}+\frac{\partial v_{0}}{\partial \vartheta}\right) \\
H_{0}(\psi)=\mu_{0} \frac{\partial \psi_{0}}{\partial \varphi}+v_{0} \frac{\partial \psi_{0}}{\partial \vartheta}+\Im \psi\left(\frac{\partial \mu_{0}}{\partial \varphi}+\frac{\partial v_{0}}{\partial \vartheta}\right) . \\
H_{1}(\mu)=\mu_{1} \frac{\partial \mu_{0}}{\partial \varphi}+\mu_{0} \frac{\partial \mu_{1}}{\partial \varphi}+v_{1} \frac{\partial \mu_{0}}{\partial \varphi}+v_{0} \frac{\partial \mu_{1}}{\partial \varphi}+\frac{1}{\omega_{0}}\left(\omega_{0} \frac{\partial \psi_{1}}{\partial \varphi}-\omega_{1} \frac{\partial \psi_{0}}{\partial \varphi}\right) \\
H_{1}(v)=\mu_{1} \frac{\partial v_{0}}{\partial \varphi}+\mu_{0} \frac{\partial v_{1}}{\partial \varphi}+v_{1} \frac{\partial v_{0}}{\partial \varphi}+v_{0} \frac{\partial v_{1}}{\partial \varphi}+\frac{1}{\omega_{0}}\left(\omega_{0} \frac{\partial \psi_{1}}{\partial \vartheta}-\omega_{1} \frac{\partial \psi_{0}}{\partial \vartheta}\right), \\
H_{1}(\omega)=\mu_{1} \frac{\partial \omega_{0}}{\partial \varphi}+\mu_{0} \frac{\partial \omega_{1}}{\partial \varphi}+v_{1} \frac{\partial \omega_{0}}{\partial \varphi}+v_{0} \frac{\partial \omega_{1}}{\partial \varphi}+\omega_{1} \frac{\partial \mu_{0}}{\partial \varphi}+\omega_{0} \frac{\partial \mu_{1}}{\partial \varphi}+\omega_{1} \frac{\partial v_{0}}{\partial \vartheta}+\omega_{0} \frac{\partial v_{1}}{\partial \vartheta} \\
H_{1}(\psi)=\mu_{1} \frac{\partial \psi_{0}}{\partial \varphi}+\mu_{0} \frac{\partial \psi_{1}}{\partial \varphi}+v_{1} \frac{\partial \psi_{0}}{\partial \varphi}+v_{0} \frac{\partial \psi_{1}}{\partial \varphi}+\psi_{1} \frac{\partial \mu_{0}}{\partial \varphi}+\psi_{0} \frac{\partial \mu_{0}}{\partial \varphi}+\psi_{1} \frac{\partial \psi_{0}}{\partial \vartheta}+\psi_{0} \frac{\partial v_{1}}{\partial \vartheta}
\end{gathered}
$$

Comparing the coefficients of same power of $p$, we have

$$
\begin{gathered}
p^{0}: \mu_{0}(\varphi, \vartheta, \Im)=e^{\varphi+\vartheta}, p^{0}: \nu_{0}(\varphi, \vartheta, \Im)=-1-e^{\varphi+\vartheta}, \\
p^{0}: \omega_{0}(\varphi, y, \Im)=e^{\varphi+\vartheta}, p^{0}: \psi_{0}(\varphi, y, \Im)=c . \\
p^{1}: \mu_{1}(\varphi, \vartheta, \Im)=-\left[E^{-1}\left\{s^{\sigma} E\left\{H_{0}(\mu)\right\}\right\}\right]=e^{\varphi+\vartheta} \frac{\Im^{\sigma}}{\Gamma(\sigma+1)}, \\
p^{1}: \nu_{1}(\varphi, \vartheta, \Im)=-\left[E^{-1}\left\{s^{\sigma} E\left\{H_{0}(\nu)\right\}\right\}\right]=-e^{\varphi+\vartheta} \frac{\Im^{\sigma}}{\Gamma(\sigma+1)}, \\
p^{1}: \omega_{1}(\varphi, \vartheta, \Im)=-\left[E^{-1}\left\{s^{\sigma} E\left\{H_{0}(\omega)\right\}\right\}\right]=e^{\varphi+\vartheta} \frac{\Im^{\sigma}}{\Gamma(\sigma+1)}, \\
p^{1}: \psi_{1}(\varphi, \vartheta, \Im)=-\left[E^{-1}\left\{s^{\sigma} E\left\{H_{0}(\psi)\right\}\right\}\right]=0,
\end{gathered}
$$




$$
\begin{aligned}
& p^{2}: \mu_{2}(\varphi, \vartheta, \Im)=-\left[E^{-1}\left\{s^{\sigma} E\left\{H_{1}(\mu)\right\}\right\}\right]=e^{\varphi+\vartheta} \frac{\Im^{2 \sigma}}{\Gamma(2 \sigma+1)}, \\
& p^{2}: v_{2}(\varphi, \vartheta, \Im)=-\left[E^{-1}\left\{s^{\sigma} E\left\{H_{1}(v)\right\}\right\}\right]=-e^{\varphi+\vartheta} \frac{\Im^{2 \sigma}}{\Gamma(2 \sigma+1)}, \\
& p^{2}: \omega_{2}(\varphi, \vartheta, \Im)=-\left[E^{-1}\left\{s^{\sigma} E\left\{H_{1}(\omega)\right\}\right\}\right]=e^{\varphi+\vartheta} \frac{\Im^{2 \sigma}}{\Gamma(2 \sigma+1)}, \\
& p^{2}: \psi_{2}(\varphi, \vartheta, \Im)=-\left[E^{-1}\left\{s^{\sigma} E\left\{H_{1}(\psi)\right\}\right\}\right]=0,
\end{aligned}
$$

The given example is a series form solution given as

$$
\begin{gathered}
\mu_{m}(\varphi, \vartheta, \Im)=e^{\varphi+\vartheta} \frac{\Im^{m \sigma}}{\Gamma(m \sigma+1)}, \quad v_{m}(\varphi, \vartheta, \Im)=-e^{\varphi+\vartheta} \frac{\Im^{m \sigma}}{\Gamma(m \sigma+1)}, \\
\omega_{m}(\varphi, \vartheta, \Im)=e^{\varphi+\vartheta} \frac{\Im^{m \sigma}}{\Gamma(m \sigma+1)}, \quad \psi_{m}(\varphi, \vartheta, 0)=0, \quad m=1,2, \cdots, \\
\mu_{m}(\varphi, \vartheta, \Im)=\mu_{0}(\varphi, \vartheta, \Im)+\mu_{1}(\varphi, \vartheta, \Im)+\mu_{2}(\varphi, \vartheta, \Im)+\mu_{3}(\varphi, \vartheta, \Im)+\cdots, \\
v_{m}(\varphi, \vartheta, \Im)=v_{0}(\varphi, \vartheta, \Im)+v_{1}(\varphi, \vartheta, \Im)+v_{2}(\varphi, \vartheta, \Im)+v_{3}(\varphi, \vartheta, \Im)+\cdots, \\
\omega_{m}(\varphi, \vartheta, \Im)=\omega_{0}(\varphi, \vartheta, \Im)+\omega_{1}(\varphi, \vartheta, \Im)+\omega_{2}(\varphi, \vartheta, \Im)+\omega_{3}(\varphi, \vartheta, \Im)+\cdots, \\
\psi_{m}(\varphi, \vartheta, 0)=\psi_{0}(\varphi, \vartheta, \Im)+\psi_{1}(\varphi, \vartheta, \Im)+\psi_{2}(\varphi, \vartheta, \Im)+\psi_{3}(\varphi, \vartheta, \Im)+\cdots, \\
\mu(\varphi, \vartheta, \Im)=e^{\varphi+\vartheta}+e^{\varphi+\vartheta} \frac{\Im^{\sigma}}{\Gamma(\sigma+1)}+e^{\varphi+\vartheta} \frac{\Im^{2 \sigma}}{\Gamma(2 \sigma+1)}+e^{\varphi+\vartheta} \frac{\Im^{3 \sigma}}{\Gamma(3 \sigma+1)}+\cdots, \\
v(\varphi, \vartheta, \Im)=-1-e^{\varphi+\vartheta}-e^{\varphi+\vartheta} \frac{\Im^{\sigma}}{\Gamma(\sigma+1)}-e^{\varphi+\vartheta} \frac{\Im^{2 \sigma}}{\Gamma(2 \sigma+1)}-e^{\varphi+\vartheta} \frac{\Im^{3 \sigma}}{\Gamma(3 \sigma+1)}-\cdots, \\
\omega(\varphi, \vartheta, \Im)=e^{\varphi+\vartheta}+e^{\varphi+\vartheta} \frac{\Im^{\sigma}}{\Gamma(\sigma+1)}+e^{\varphi+\vartheta} \frac{\Im^{2 \sigma}}{\Gamma(2 \sigma+1)}+e^{\varphi+\vartheta} \frac{\Im^{3 \sigma}}{\Gamma(3 \sigma+1)}+\cdots, \\
\psi(\varphi, \vartheta, \Im)=c+0+\cdots, \quad
\end{gathered}
$$

The approximate solution by VITM.

According to the iteration method for the scheme (18), we have

$$
\begin{aligned}
& \mu_{m+1}(\varphi, \vartheta, \Im)=\mu_{m}(\varphi, \vartheta, \Im)-E^{-1}\left[s^{\sigma} E\left\{\frac{1}{s^{\sigma}} \frac{\partial \mu_{m}}{\partial \Im}+\mu_{m} \frac{\partial \mu_{m}}{\partial \varphi}+v_{m} \frac{\partial \mu_{m}}{\partial \vartheta}+\frac{1}{\omega_{m}} \frac{\partial \psi_{m}}{\partial \varphi}\right\}\right], \\
& v_{m+1}(\varphi, \vartheta, \Im)=v_{m}(\varphi, \vartheta, \Im)-E^{-1}\left[s^{\sigma} E\left\{\frac{1}{s^{\sigma}} \frac{\partial v_{m}}{\partial \Im}+\mu_{m} \frac{\partial v_{m}}{\partial \varphi}+v_{m} \frac{\partial v_{m}}{\partial \vartheta}+\frac{1}{\omega_{m}} \frac{\partial \psi_{m}}{\partial \vartheta}\right\}\right], \\
& \omega_{m+1}(\varphi, \vartheta, \Im)=\omega_{m}(\varphi, \vartheta, \Im)-E^{-1}\left[s^{\sigma} E\left\{\frac{1}{s^{\sigma}} \frac{\partial \mu_{m}}{\partial \Im}+\mu_{m} \frac{\partial \omega_{m}}{\partial \varphi}+v_{m} \frac{\partial \omega_{m}}{\partial \vartheta}+\omega_{m}\left(\frac{\partial \mu_{m}}{\partial \varphi}+\frac{\partial v_{m}}{\partial \vartheta}\right)\right\}\right], \\
& \psi_{m+1}(\varphi, \vartheta, \Im)=\psi_{m}(\varphi, \vartheta, \Im)-E^{-1}\left[s^{\sigma} E\left\{\frac{1}{s^{\sigma}} \frac{\partial v_{m}}{\partial \Im}+\mu_{m} \frac{\partial \psi_{m}}{\partial \varphi}+v_{m} \frac{\partial \psi_{m}}{\partial \vartheta}+\Im \psi_{m}\left(\frac{\partial \mu_{m}}{\partial \varphi}+\frac{\partial v_{m}}{\partial \vartheta}\right)\right\}\right],
\end{aligned}
$$

with the initial sources

$$
\begin{aligned}
& \mu_{0}(\varphi, \vartheta, \Im)=e^{\varphi+\vartheta}, \quad \nu_{0}(\varphi, \vartheta, \Im)=-1-e^{\varphi+\vartheta}, \\
& \omega_{0}(\varphi, \vartheta, \Im)=e^{\varphi+\vartheta}, \quad \psi_{0}(\varphi, \vartheta, 0)=c .
\end{aligned}
$$

For $m=0,1,2, \cdots$

$$
\mu_{1}(\varphi, \vartheta, \Im)=\mu_{0}(\varphi, \vartheta, \Im)-E^{-1}\left[s^{\sigma} E\left\{\frac{1}{s^{\sigma}} \frac{\partial \mu_{0}}{\partial \Im}+\mu_{0} \frac{\partial \mu_{0}}{\partial \varphi}+v_{0} \frac{\partial \mu_{0}}{\partial \vartheta}+\frac{1}{\omega_{0}} \frac{\partial \psi_{0}}{\partial \varphi}\right\}\right],
$$




$$
\begin{aligned}
& v_{1}(\varphi, \vartheta, \Im)=v_{0}(\varphi, \vartheta, \Im)-E^{-1}\left[s^{\sigma} E\left\{\frac{1}{s^{\sigma}} \frac{\partial v_{0}}{\partial \Im}+\mu_{0} \frac{\partial v_{0}}{\partial \varphi}+v_{0} \frac{\partial v_{0}}{\partial \vartheta}+\frac{1}{\omega_{0}} \frac{\partial \psi_{0}}{\partial \vartheta}\right\}\right], \\
& \omega_{1}(\varphi, \vartheta, \Im)=\omega_{0}(\varphi, \vartheta, \Im)-E^{-1}\left[s^{\sigma} E\left\{\frac{1}{s^{\sigma}} \frac{\partial \mu_{0}}{\partial \Im}+\mu_{0} \frac{\partial \omega_{0}}{\partial \varphi_{0}}+v_{0} \frac{\partial \omega_{0}}{\partial \vartheta_{0}}+\omega_{0}\left(\frac{\partial \mu_{0}}{\partial \varphi}+\frac{\partial v_{0}}{\partial \vartheta}\right)\right\}\right], \\
& \psi_{1}(\varphi, \vartheta, \Im)=\psi_{0}(\varphi, \vartheta, \Im)-E^{-1}\left[s^{\sigma} E\left\{\frac{1}{s^{\sigma}} \frac{\partial v_{0}}{\partial \Im}+\mu_{0} \frac{\partial \psi_{0}}{\partial \varphi}+v_{0} \frac{\partial \psi_{0}}{\partial \vartheta}+\Im \psi_{0}\left(\frac{\partial \mu_{0}}{\partial \varphi}+\frac{\partial v_{0}}{\partial \vartheta}\right)\right\}\right], \\
& \mu_{1}(\varphi, \vartheta, \Im)=e^{\varphi+\vartheta}\left\{1+\frac{\Im^{\sigma}}{\Gamma(\sigma+1)}\right\}, \quad v_{1}(\varphi, \vartheta, \Im)=-1-e^{\varphi+\vartheta}\left\{1+\frac{\Im^{\sigma}}{\Gamma(\sigma+1)}\right\}, \\
& \omega_{1}(\varphi, \vartheta, \Im)=e^{\varphi+\vartheta}\left\{1+\frac{\Im^{\sigma}}{\Gamma(\sigma+1)}\right\}, \quad \psi_{1}(\varphi, \vartheta, 0)=c+0 . \\
& \mu_{2}(\varphi, \vartheta, \Im)=\mu_{1}(\varphi, \vartheta, \Im)-E^{-1}\left[s^{\sigma} E\left\{\frac{1}{s^{\sigma}} \frac{\partial \mu_{1}}{\partial \Im}+\mu_{1} \frac{\partial \mu_{1}}{\partial \varphi}+v_{1} \frac{\partial \mu_{1}}{\partial \vartheta}+\frac{1}{\omega_{1}} \frac{\partial \psi_{1}}{\partial \varphi}\right\}\right], \\
& v_{2}(\varphi, \vartheta, \Im)=v_{1}(\varphi, \vartheta, \Im)-E^{-1}\left[s^{\sigma} E\left\{\frac{1}{s^{\sigma}} \frac{\partial v_{1}}{\partial \Im}+\mu_{1} \frac{\partial v_{1}}{\partial \varphi}+v_{1} \frac{\partial v_{1}}{\partial \vartheta}+\frac{1}{\omega_{1}} \frac{\partial \psi_{1}}{\partial \vartheta}\right\}\right], \\
& \omega_{2}(\varphi, \vartheta, \Im)=\omega_{1}(\varphi, \vartheta, \Im)-E^{-1}\left[s^{\sigma} E\left\{\frac{1}{s^{\sigma}} \frac{\partial \mu_{1}}{\partial \Im}+\mu_{1} \frac{\partial \omega_{1}}{\partial \varphi_{0}}+v_{1} \frac{\partial \omega_{1}}{\partial \vartheta_{1}}+\omega_{1}\left(\frac{\partial \mu_{1}}{\partial \varphi}+\frac{\partial v_{1}}{\partial \vartheta}\right)\right\}\right], \\
& \psi_{2}(\varphi, \vartheta, \Im)=\psi_{1}(\varphi, \vartheta, \Im)-E^{-1}\left[s^{\sigma} E\left\{\frac{1}{s^{\sigma}} \frac{\partial \nu_{1}}{\partial \Im}+\mu_{1} \frac{\partial \psi_{1}}{\partial \varphi}+v_{1} \frac{\partial \psi_{1}}{\partial \vartheta}+\Im \psi_{1}\left(\frac{\partial \mu_{1}}{\partial \varphi}+\frac{\partial \nu_{1}}{\partial \vartheta}\right)\right\}\right], \\
& \mu_{2}(\varphi, \vartheta, \Im)=e^{\varphi+\vartheta}\left\{1+\frac{\Im^{\sigma}}{\Gamma(\sigma+1)}+\frac{\Im^{2 \sigma}}{\Gamma(2 \sigma+1)}\right\}, \\
& v_{2}(\varphi, \vartheta, \Im)=-1-e^{\varphi+\vartheta}\left\{1+\frac{\Im^{\sigma}}{\Gamma(\sigma+1)}+\frac{\Im^{2 \sigma}}{\Gamma(2 \sigma+1)}\right\}, \\
& \omega_{2}(\varphi, \vartheta, \Im)=e^{\varphi+\vartheta}\left\{1+\frac{\Im^{\sigma}}{\Gamma(\sigma+1)}+\frac{\Im^{2 \sigma}}{\Gamma(2 \sigma+1)}\right\}, \\
& \psi_{2}(\varphi, \vartheta, 0)=c+0 \text {. } \\
& \mu_{3}(\varphi, \vartheta, \Im)=\mu_{2}(\varphi, \vartheta, \Im)-E^{-1}\left[s^{\sigma} E\left\{\frac{1}{s^{\sigma}} \frac{\partial \mu_{2}}{\partial \Im}+\mu_{2} \frac{\partial \mu_{2}}{\partial \varphi}+v_{2} \frac{\partial \mu_{2}}{\partial \vartheta}+\frac{1}{\omega_{2}} \frac{\partial \psi_{2}}{\partial \varphi}\right\}\right], \\
& v_{3}(\varphi, \vartheta, \Im)=v_{2}(\varphi, \vartheta, \Im)-E^{-1}\left[s^{\sigma} E\left\{\frac{1}{s^{\sigma}} \frac{\partial v_{2}}{\partial \Im}+\mu_{2} \frac{\partial v_{2}}{\partial \varphi}+v_{2} \frac{\partial v_{2}}{\partial \vartheta}+\frac{1}{\omega_{2}} \frac{\partial \psi_{2}}{\partial \vartheta}\right\}\right], \\
& \omega_{3}(\varphi, \vartheta, \Im)=\omega_{2}(\varphi, \vartheta, \Im)-E^{-1}\left[s^{\sigma} E\left\{\frac{1}{s^{\sigma}} \frac{\partial \mu_{2}}{\partial \Im}+\mu_{2} \frac{\partial \omega_{2}}{\partial \varphi_{0}}+v_{2} \frac{\partial \omega_{2}}{\partial \vartheta_{2}}+\omega_{2}\left(\frac{\partial \mu_{2}}{\partial \varphi}+\frac{\partial v_{2}}{\partial \vartheta}\right)\right\}\right], \\
& \psi_{3}(\varphi, \vartheta, \Im)=\psi_{2}(\varphi, \vartheta, \Im)-E^{-1}\left[s^{\sigma} E\left\{\frac{1}{s^{\sigma}} \frac{\partial v_{2}}{\partial \Im}+\mu_{2} \frac{\partial \psi_{2}}{\partial \varphi}+v_{2} \frac{\partial \psi_{2}}{\partial \vartheta}+\Im \psi_{2}\left(\frac{\partial \mu_{2}}{\partial \varphi}+\frac{\partial v_{2}}{\partial \vartheta}\right)\right\}\right], \\
& \mu_{3}(\varphi, \vartheta, \Im)=e^{\varphi+\vartheta}\left\{1+\frac{\Im^{\sigma}}{\Gamma(\sigma+1)}+\frac{\Im^{2 \sigma}}{\Gamma(2 \sigma+1)}+\frac{\Im^{3 \sigma}}{\Gamma(3 \sigma+1)}\right\}, \\
& v_{3}(\varphi, \vartheta, \Im)=-1-e^{\varphi+\vartheta}\left\{1+\frac{\Im^{\sigma}}{\Gamma(\sigma+1)}+\frac{\Im^{2 \sigma}}{\Gamma(2 \sigma+1)}+\frac{\Im^{3 \sigma}}{\Gamma(3 \sigma+1)}\right\}, \\
& \omega_{3}(\varphi, \vartheta, \Im)=e^{\varphi+\vartheta}\left\{1+\frac{\Im^{\sigma}}{\Gamma(\sigma+1)}+\frac{\Im^{2 \sigma}}{\Gamma(2 \sigma+1)}+\frac{\Im^{3 \sigma}}{\Gamma(3 \sigma+1)}\right\}, \\
& \psi_{3}(\varphi, \vartheta, \Im)=c+0 .
\end{aligned}
$$$$
\mu(\varphi, \vartheta, \Im)=\sum_{m=0}^{\infty} \mu_{m}(\varphi, \vartheta, \Im)=e^{\varphi+\vartheta}\left\{1+\frac{\Im^{\sigma}}{\Gamma(\sigma+1)}+\frac{\Im^{2 \sigma}}{\Gamma(2 \sigma+1)}+\frac{\Im^{3 \sigma}}{\Gamma(3 \sigma+1)}+\cdots+\frac{\Im^{m \sigma}}{\Gamma(m \sigma+1)}\right\},
$$ 


$$
\begin{array}{r}
v(\varphi, \vartheta, \Im)=\sum_{m=0}^{\infty} v_{m}(\varphi, \vartheta, \Im)=-1-e^{\varphi+\vartheta}\left\{1+\frac{\Im^{\sigma}}{\Gamma(\sigma+1)}+\frac{\Im^{2 \sigma}}{\Gamma(2 \sigma+1)}+\frac{\Im^{3 \sigma}}{\Gamma(3 \sigma+1)}+\cdots+\frac{\Im^{m \sigma}}{\Gamma(m \sigma+1)}\right\} \\
\omega(\varphi, \vartheta, \Im)=\sum_{m=0}^{\infty} \omega_{m}(\varphi, \vartheta, \Im)=e^{\varphi+\vartheta}\left\{1+\frac{\Im^{\sigma}}{\Gamma(\sigma+1)}+\frac{\Im^{2 \sigma}}{\Gamma(2 \sigma+1)}+\frac{\Im^{3 \sigma}}{\Gamma(3 \sigma+1)}+\cdots+\frac{\Im^{m \sigma}}{\Gamma(m \sigma+1)}\right\} \\
\psi(\varphi, \vartheta, \Im)=\sum_{m=0}^{\infty} \psi_{m}(\varphi, \vartheta, \Im)=c+0 .
\end{array}
$$

The exact result of Equation (22) at $\sigma=1$,

$$
\begin{aligned}
& \mu(\varphi, \vartheta, \Im)=e^{\varphi+\vartheta+\Im}, \quad \nu(\varphi, \vartheta, \Im)=-1-e^{\varphi+\vartheta+\Im} \\
& \omega(\varphi, \vartheta, \Im)=e^{\varphi+\vartheta+\Im}, \quad \psi(\varphi, \vartheta, \Im)=c .
\end{aligned}
$$

\section{Discussion and Numerical Results}

In this section, we discuss the numerical analysis of the obtained solutions for four differential equation schemes discussing the unsteady flow of a polytropical gas of arbitrary order. Figures 1 and 2 shows results of two-dimensional plots of actual and HPTM solutions for $\varphi \in[0 ; 1], \Im=0.1$ and $\vartheta=1$. In Figures $3-8$ the 3D graph of $\mu(\varphi, \vartheta, \Im)$, $\nu(\varphi, \vartheta, \Im)$ and $\omega(\varphi, \vartheta, \Im)$ for various fractional order are presented, which shows that the derived HPTM/VITM approximated results are in strong agreement with the exact and the numerical ones. This comparison represents a strong correlation between the HPTM/VITM and exact findings. Figures 9 and 10 shows results of two-dimensional plots of actual and VITM solutions for $\varphi \in[0 ; 1], \Im=0.1$ and $\vartheta=1$. Figures 11 and 12 depicts solutions in two-dimensional plots for different values of $\sigma=1,0.9,0.8,0.7,0.6,0.5$ for $\varphi \in[0 ; 1]$, $\Im$ and $\vartheta=1$. The same figures of the suggested techniques are obtained and the validity of the proposed methods has been verified. Numerical evaluations were performed to ensure that the proposed methods are simple and effective.
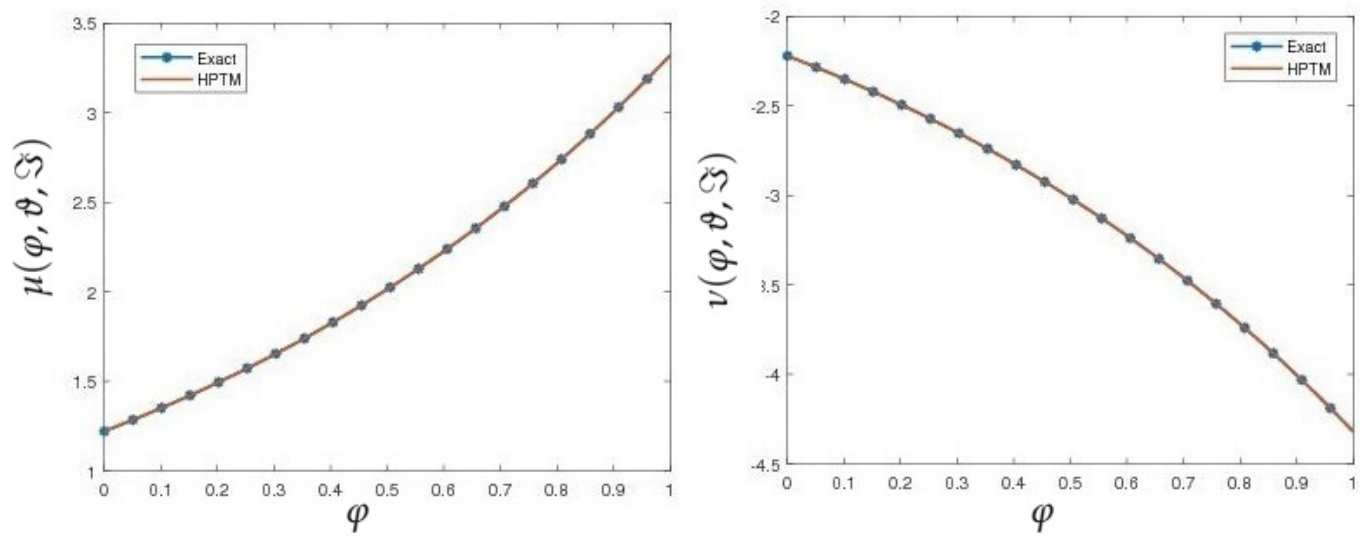

Figure 1. The exact and approximate solution graphs of $\mu(\varphi, \vartheta)$ and $\nu(\varphi, \vartheta)$, Example 1. 

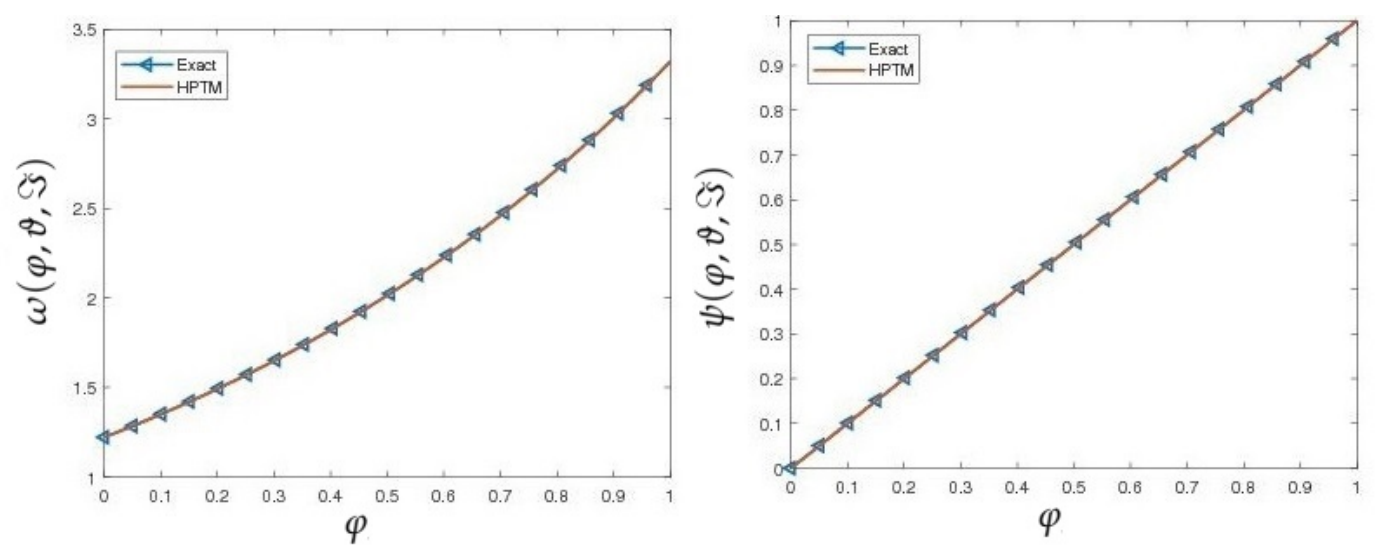

Figure 2. The exact and approximate solution graphs of $\omega(\varphi, \vartheta)$ and $\psi(\varphi, \vartheta)$, Example 1 .
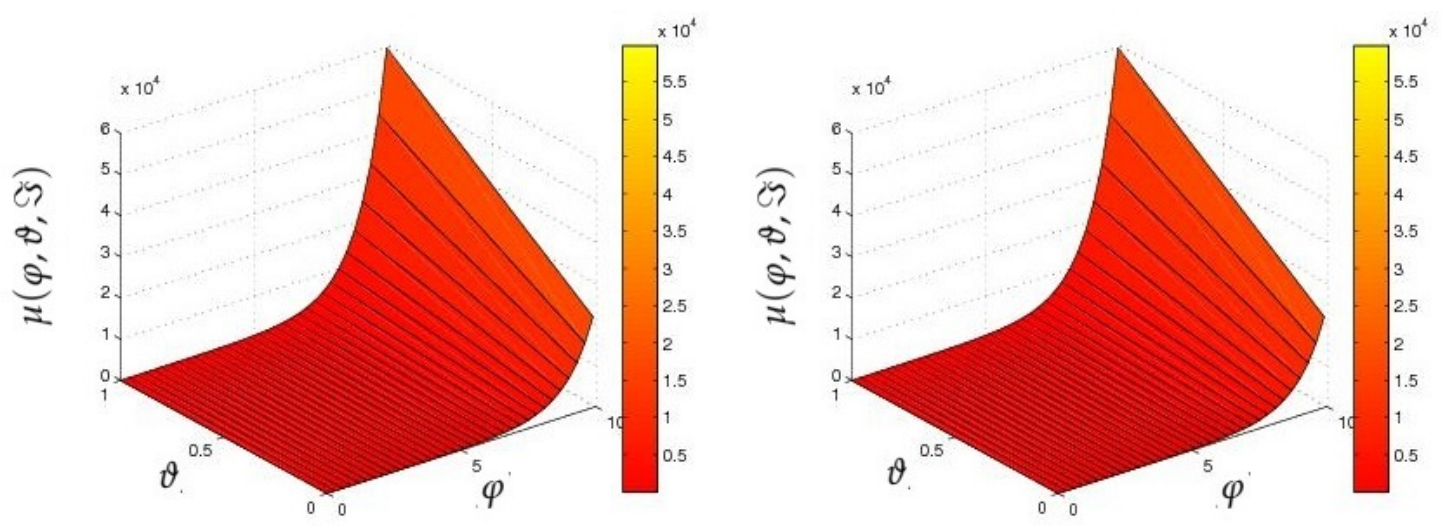

Figure 3. The HPTM graphs of $\delta=1$ and 0.8 of $\mu(\varphi, \vartheta)$, Example 1 .
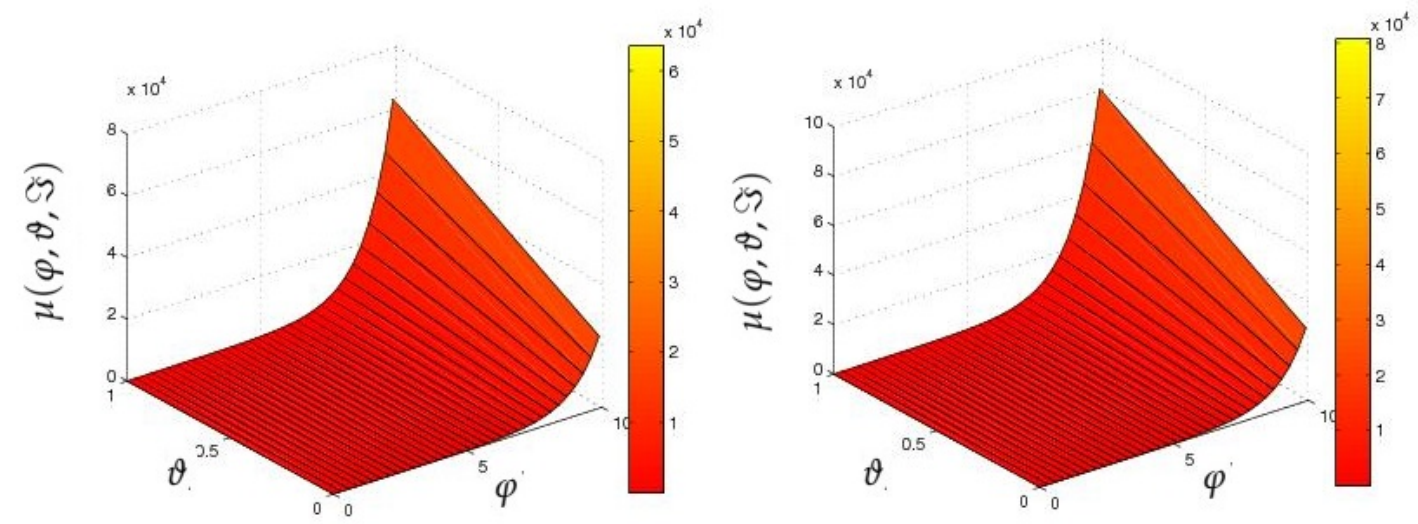

Figure 4. The HPTM graphs of $\delta=0.6$ and 0.4 of $\mu(\varphi, \vartheta)$, Example 1 . 

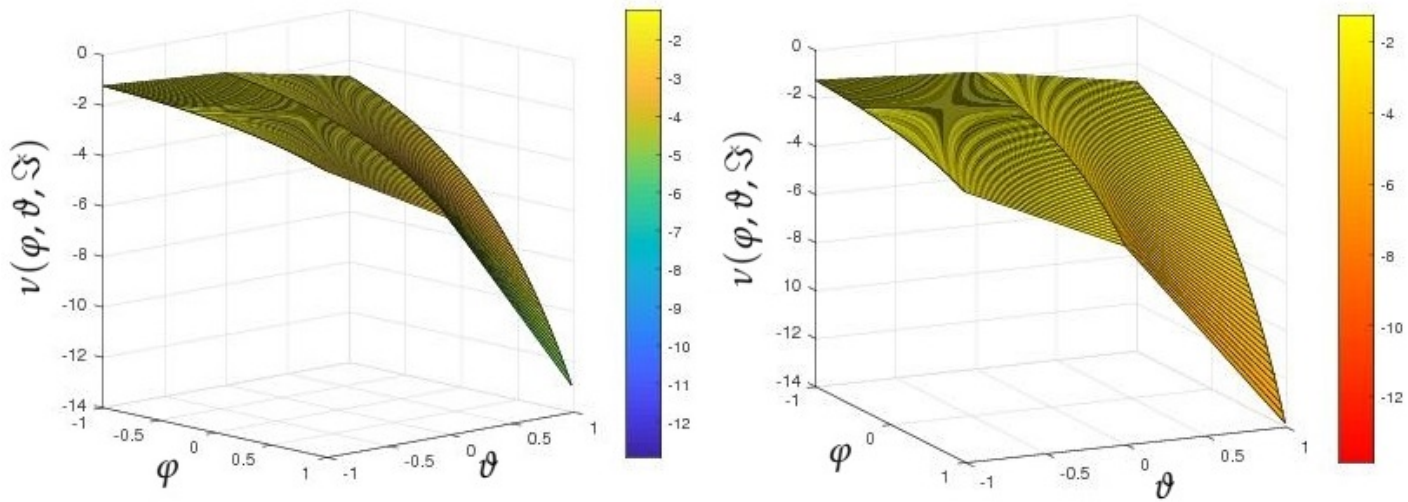

Figure 5. The HPTM graphs of $\delta=1$ and 0.8 of $\nu(\varphi, \vartheta)$ Example 1.
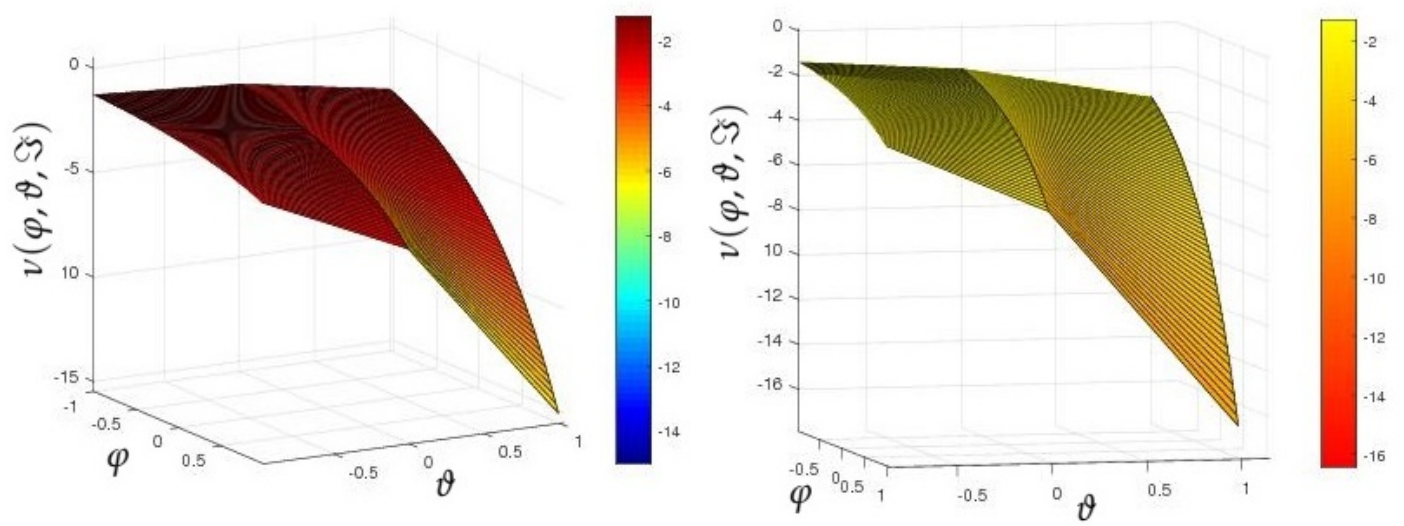

Figure 6. The HPTM graphs of $\delta=0.6$ and 0.4 of $v(\varphi, \vartheta)$, Example 1 .
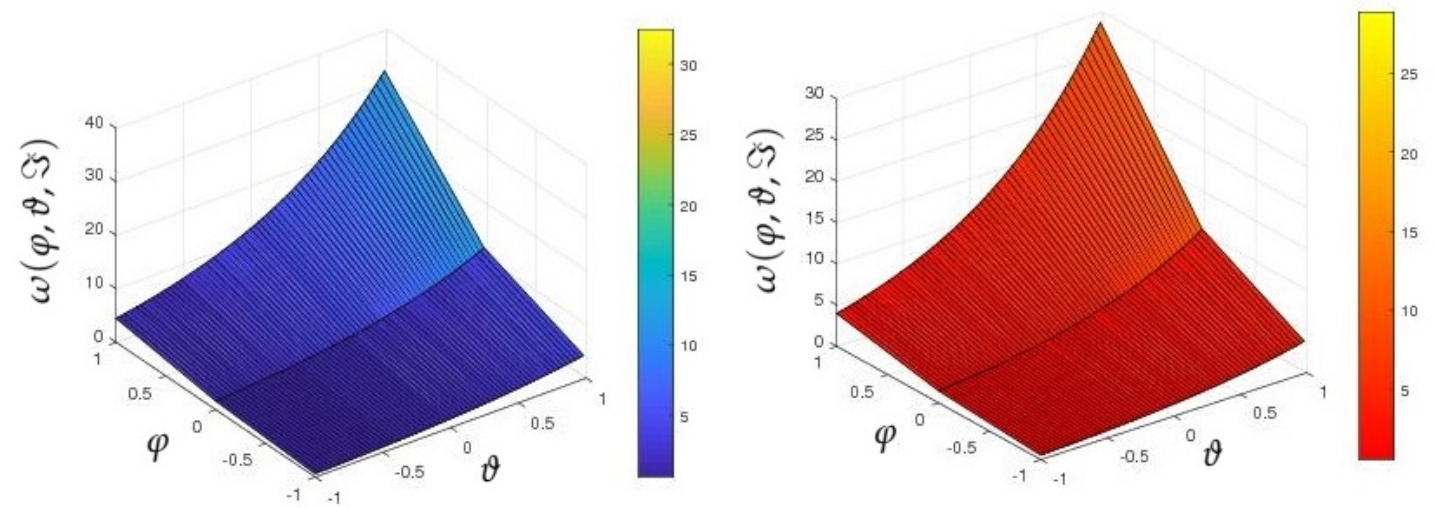

Figure 7. The HPTM graphs of $\delta=1$ and 0.8 of $\omega(\varphi, \vartheta)$, Example 1. 

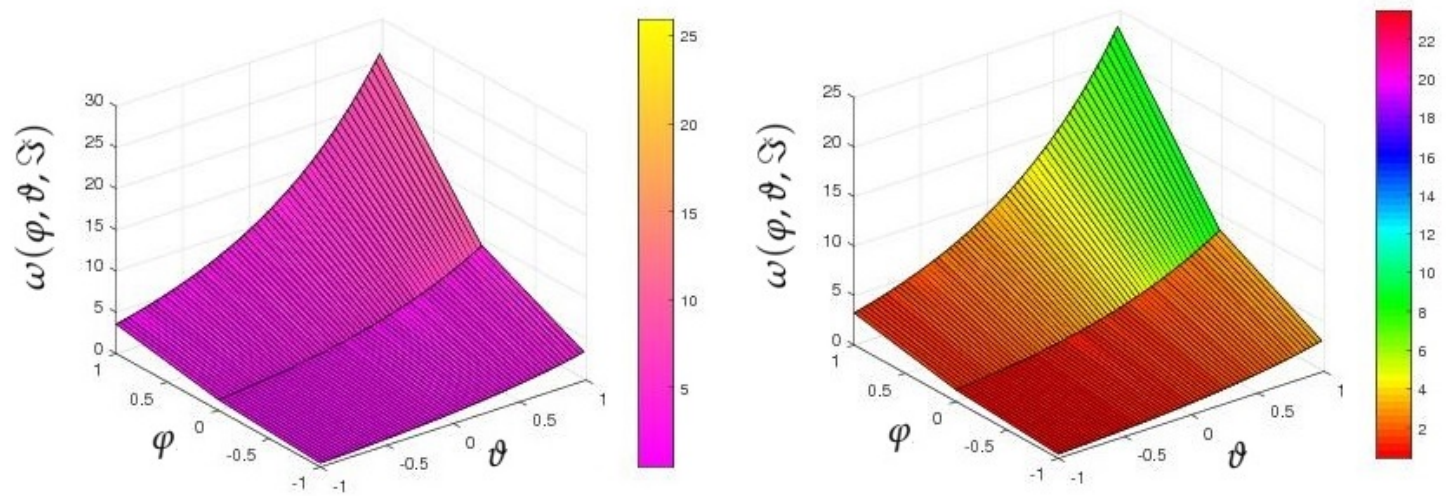

Figure 8. The HPTM graphs of $\delta=0.6$ and 0.4 of $\omega(\varphi, \vartheta)$, Example 1 .
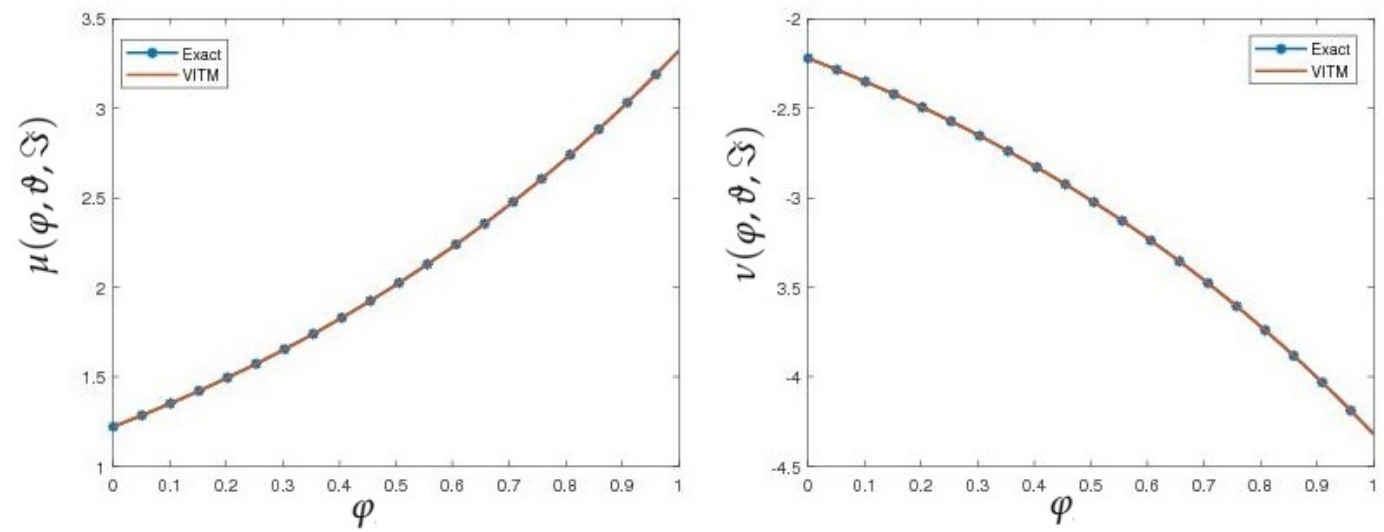

Figure 9. The exact and approximate solution graphs of $\mu(\varphi, \vartheta)$ and $\nu(\varphi, \vartheta)$, Example 1.
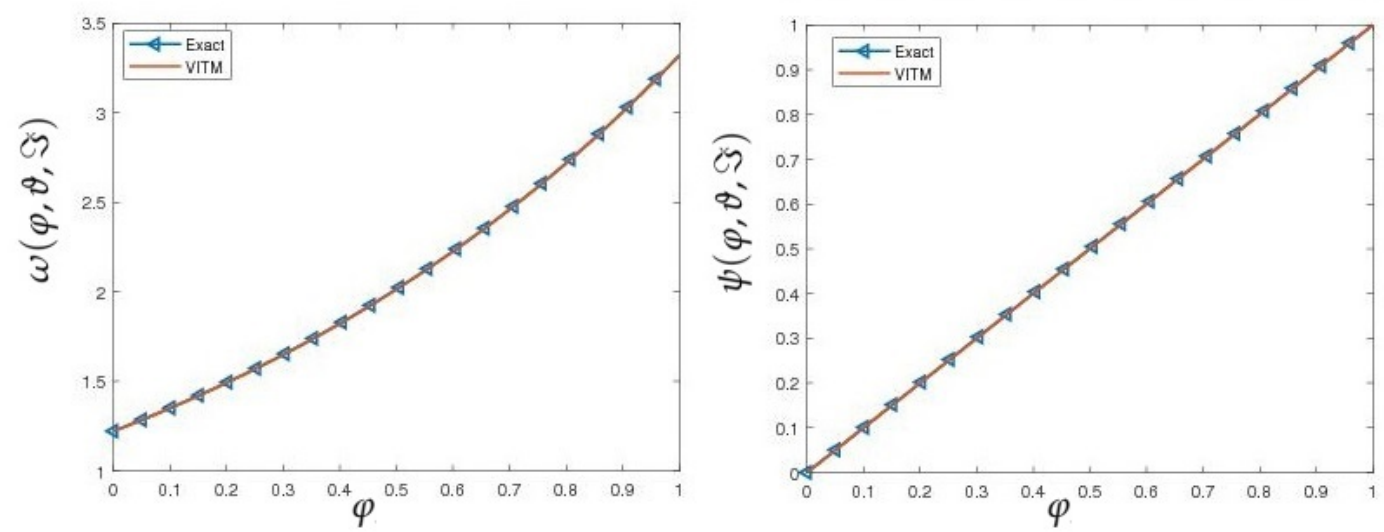

Figure 10. The exact and approximate solution graphs of $\omega(\varphi, \vartheta)$ and $\psi(\varphi, \vartheta)$, Example 1. 

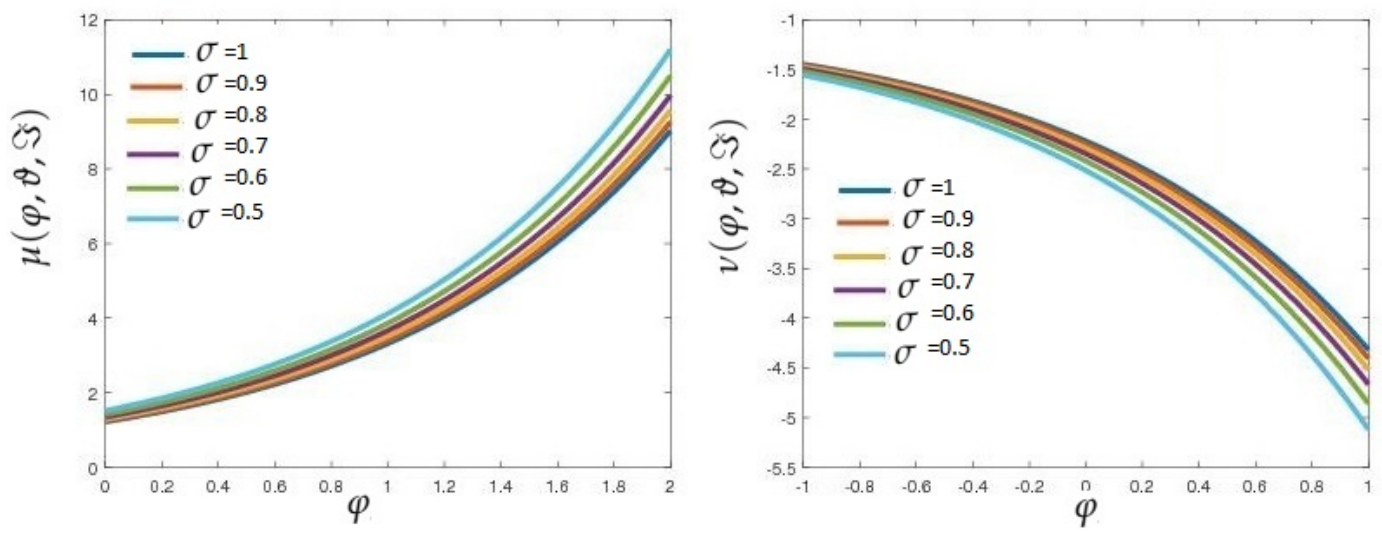

Figure 11. The approximate solution graphs of different fractional order of $\delta$ for $\mu(\varphi, \vartheta)$ and $\nu(\varphi, \vartheta)$, Example 1 .

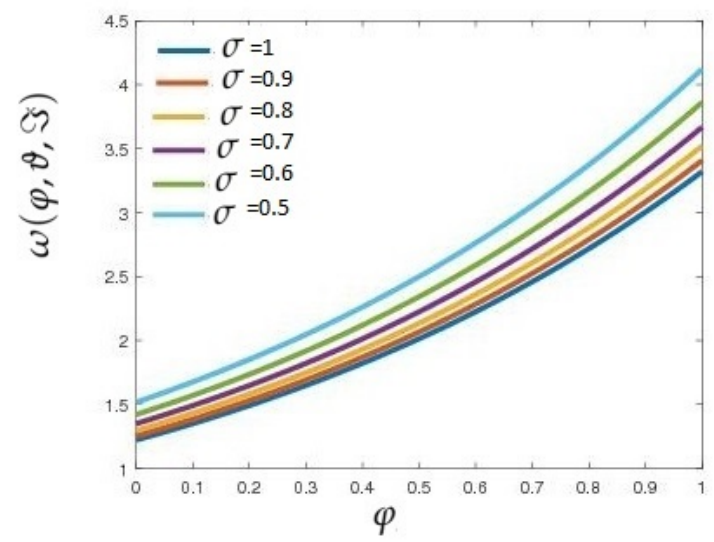

Figure 12. The approximate solution graph of different fractional order of $\delta$ for $\omega(\varphi, \vartheta)$.

\section{Conclusions}

In this article, two semi-analytical techniques are implemented to solve fractionalorder gas dynamics equations. The approximate solution of the system is evaluated to confirm the validity and reliability of the proposed methods. Graphs of the solutions are plotted to display the close relation between the obtained and exact results. The problem shows this to validate and test the efficacy of the suggested techniques. Moreover, the efficiency of the mentioned procedures and the reduction in calculations provides broader applicability. It is also illustrated that the findings of the current methodology are close to the exact solutions. The proposed methods are powerful techniques to solve other nonlinear systems of fractional-order partial differential equations.

Author Contributions: Data curation, K.N.; Formal analysis, S.-S.Z.; Methodology, S.-S.Z. and S.S.; Project administration, N.-A.S.; Software, S.S.; Validation, K.N.; Writing-original draft, N.A.S.; Writing-review editing, I.D. All authors have read and agreed to the published version of the manuscript.

Funding: This research received no external funding.

Institutional Review Board Statement: Not applicable.

Informed Consent Statement: Not applicable.

Data Availability Statement: Not applicable.

Acknowledgments: The authors extend their appreciation to the Deanship of Scientific Research at King Khalid University for funding this work through research groups program under Grant No. RGP.1/46/42. This work was supported by the Scientific Research Fund of Hunan Provincial Education Department (Grant No.: 19A092), and Key Laboratory of Key Technologies of Digital Urban-Rural Spatial Planning of Hunan Province (Grant No.: 2018TP1042). 
Conflicts of Interest: The authors have no conflict of interest.

\section{References}

1. Shah, K.; Singh, T.; Kilin, A. Combination of integral and projected differential transform methods for time-fractional gas dynamics equations. Ain Shams Eng. J. 2018, 9, 1683-1688. [CrossRef]

2. Steger, J.L.; Warming, R.F. Flux vector splitting of the inviscid gasdynamic equations with application to finite-difference methods. J. Comput. Phys. 1981, 40, 263-293. [CrossRef]

3. Kumar, S.; Kocak, H.; Yildirim, A. A fractional model of gas dynamics equations and its analytical approximate solution using Laplace transform. Z. Naturforschung A 2012, 67, 389-396. [CrossRef]

4. Zaslavsky, G.M.; Edelman, M. Hierarchical structures in the phase space and fractional kinetics: I. Classical systems. Chaos Interdiscip. J. Nonlinear Sci. 2000, 10, 135-146. [CrossRef]

5. Uchaikin, V.V.; Sibatov, R.T. Fractional Boltzmann equation for multiple scattering of resonance radiation in low-temperature plasma. J. Phys. Math. Theor. 2011, 44, 145501. [CrossRef]

6. Nikan, O.; Machado, J.T.; Avazzadeh, Z.; Jafari, H. Numerical evaluation of fractional Tricomi-type model arising from physical problems of gas dynamics. J. Adv. Res. 2020, 25, 205-216. [CrossRef]

7. Frenk, C.S.; White, S.D.M.; Bode, P.; Bond, J.R.; Bryan, G.L.; Cen, R.; Couchman, H.M.P.; Evrard, A.E.; Gnedin, N.; Jenkins, A.; et al. The Santa Barbara cluster comparison project: a comparison of cosmological hydrodynamics solutions. Astrophy. J. 1999, 525, 554-582. [CrossRef]

8. Ryu, D.; Ostriker, J.P.; Kang, H.; Cen, R. A cosmological hydrodynamic code based on the total variation diminishing scheme. Astrophy. J. 1993, 414, 1-19. [CrossRef]

9. Xu, K.; Luo, J.; Chen, S. A well-balanced kinetic scheme for gas dynamic equations under gravitational field. Adv. Appl. Math. Mech. 2010, 2, 200-210. [CrossRef]

10. Veeresha, P.; Prakasha, D.G.; Baskonus, H.M. An efficient technique for a fractional-order system of equations describing the unsteady flow of a polytropic gas. Pramana 2019, 93, 75. [CrossRef]

11. Moradpour, H.; Abri, A.; Ebadi, H. Thermodynamic behavior and stability of Polytropic gas. Int. J. Mod. Phys. D 2016, 25, 1650014. [CrossRef]

12. Sunthrayuth, P.; Zidan, A.; Yao, S.; Shah, R.; Inc, M. The Comparative Study for Solving Fractional-Order Fornberg-Whitham Equation via $\rho$-Laplace Transform. Symmetry 2021, 13, 784. [CrossRef]

13. Tamsir, M.; Srivastava, V.K. Revisiting the approximate analytical solution of fractional-order gas dynamics equation. Alex. Eng. J. 2016, 55, 867-874. [CrossRef]

14. Bhadane, P.K.G.; Pradhan, V.H. Elzaki transform homotopy perturbation method for solving Gas Dynamics equation. Int. J. Res. Eng. Tech. 2013, 2, 260-264.

15. Mohamed, M.A. Adomian decomposition method for solving the equation governing the unsteady flow of a polytropic gas. Appl. Math. 2009, 4, 52-61.

16. Matinfar, M.; Saeidy, M. Homotopy Analysis Method for Solving the Equation Governing the Unsteady Flow of a Polytropic Gas. World Appl. Sci. J. 2010. 9, 980-983.

17. Cherif, M.H.; Ziane, D.; Belghaba, K. Fractional natural decomposition method for solving fractional system of nonlinear equations of unsteady flow of a polytropic gas. Nonlinear Stud. 2018. 25, 753-764.

18. He, J.H. Homotopy perturbation method: a new nonlinear analytical technique. Appl. Math. Comput. 2003, 135, 73-79. [CrossRef]

19. He, J.H. Homotopy perturbation method for bifurcation of nonlinear problems. Int. J. Nonlinear Sci. Numer. Simul. 2005, 6, 207-208. [CrossRef]

20. He, J.H. Homotopy perturbation method for solving boundary value problems. Phys. Lett. A 2006. 350, 87-88. [CrossRef]

21. He, J.H. The homotopy perturbation method for nonlinear oscillators with discontinuities. Appl. Math. Comput. 2004, 151, 287-292. [CrossRef]

22. He, J.H. Application of homotopy perturbation method to nonlinear wave equations. Chaos Solitons Fractals 2005, 26, 695-700. [CrossRef]

23. Elzaki, T.M. The new integral transform Elzaki transform. Glob. J. Pure Appl. Math. 2011, 7, 57-64.

24. Elzaki, T.M. On the connections between Laplace and Elzaki transforms. Adv. Theor. Appl. Math. 2011, 6, 1-11.

25. Elzaki, T.M. On The New Integral Transform "Elzaki Transform" Fundamental Properties Investigations and Applications. Glob. J. Math. Sci. Theory Pract. 2012, 4, 1-13.

26. Jena, R.M.; Chakraverty, S. Solving time-fractional Navier-Stokes equations using homotopy perturbation Elzaki transform. SN Appl. Sci. 2019, 1, 16. [CrossRef]

27. Sedeeg, A.K.H. A coupling Elzaki transform and homotopy perturbation method for solving nonlinear fractional heat-like equations. Am. J. Math. Comput. Model. 2016, 1, 15-20.

28. Neamaty, A.; Agheli, B.; Darzi, R. Applications of homotopy perturbation method and Elzaki transform for solving nonlinear partial differential equations of fractional order. J. Nonlin. Evolut. Equat. Appl. 2016, 2015, 91-104.

29. Odibat, Z.M. A study on the convergence of variational iteration method. Math. Comput. Model. 2010, 51, 1181-1192. [CrossRef]

30. Zedan, H.A.; Tantawy, S.S.; Sayed, Y.M. Convergence of the variational iteration method for initial-boundary value problem of fractional integrodifferential equations. J. Fract. Calc. Appl. 2014, 5 (Suppl. 3), 1-14. 